\title{
Comparison of Cytologic Characteristics between Adenoid Cystic Carcinoma and Adenoid Basal Carcinoma in the Uterine Cervix
}

Juhyeon Jeong · Seung Yeon $\mathrm{Ha}$ Hyun Yee Cho · Dong Hae Chung Jungsuk An

Department of Pathology, Gachon University Gil Medical Center, Incheon, Korea

Received: May 20, 2015

Revised: June 26, 2015

Accepted: July 8, 2015

\section{Corresponding Author}

Seung Yeon $\mathrm{Ha}, \mathrm{MD}, \mathrm{PhD}$

Department of Pathology, Gachon University

Gil Medical Center, 21 Namdong-daero

774beon-gil, Namdong-gu, Incheon 21565, Korea

Tel: +82-32-460-3073

Fax: +82-32-460-2394

E-mail: syha@gilhospital.com

\begin{abstract}
Background: Adenoid cystic carcinoma (ACC) and adenoid basal carcinoma (ABC) are rare in the uterine cervix. $A C C$ is more aggressive than $A B C$, thus accurate differential diagnosis is important. In this study, we identified cytologic features useful in distinguishing these two tumors for diagnosis. Methods: Three cases of ACC and five cases of ABC were selected for this study. Cervicovaginal smear slides were reviewed retrospectively, and the area, circumference, major axis, and minor axis of nuclei were measured using an image analyzer. Results: ACC displayed threedimensional clusters with a small acini pattern. $\mathrm{ABC}$ displayed peripheral palisading without an acini pattern. The nuclei of $A C C$ were more irregular and angulated than those of $A B C$, and the former showed a coarsely granular chromatin pattern. The nucleic area, circumference, major axis, and minor axis were $18.556 \pm 8.665 \mu \mathrm{m}^{2}, 23.320 \pm 11.412 \mu \mathrm{m}, 5.664 \pm 1.537 \mu \mathrm{m}$, and $4.127 \pm$ $1.107 \mu \mathrm{m}$ in $\mathrm{ACC}$ and $11.017 \pm 4.440 \mu \mathrm{m}^{2}, 15.920 \pm 5.664 \mu \mathrm{m}, 4.612 \pm 1.025 \mu \mathrm{m}$, and $3.088 \pm 0.762$ $\mu \mathrm{m}$ in the cases of ABC. All measured values showed statistically significant difference $(p<.001)$. Conclusions: Although the nuclei of both of these tumor types were oval shaped, inferred from the ratio of minor axis to major axis (0.728 in ACC and 0.669 in $A B C)$, the area of nuclei was approximately 1.7 times larger in ACC than in ABC. Distinguishing nucleic features, including area, morphology, and chromatin pattern, may be helpful in making a correct diagnosis.
\end{abstract}

Key Words: Adenoid cystic carcinoma; Adenoid basal carcinoma; Vaginal smears; Papanicolaou test
The World Health Organization (WHO) classifies malignant tumors of the uterine cervix into three categories: squamous tumors, glandular tumors, and "other epithelial tumors." The category of "other epithelial tumors" is composed of five different tumor types: adenosquamous carcinoma, adenoid cystic carcinoma (ACC), adenoid basal carcinoma $(A B C)$, neuroendocrine tumors, and undifferentiated carcinoma. ${ }^{1}$ Both $\mathrm{ACC}$ and $\mathrm{ABC}$ are rare tumors, accounting for less than $1 \%$ of all cervical adenocarcinomas and are usually found in postmenopausal women over the age of $60 .{ }^{2}$ In spite of the fact that the cytologic features of these two malignant tumors of the uterine cervix can be very confusing, an accurate diagnosis of ACCs and ABCs is important because their clinical and biological behaviors are quite different; ACCs have worse prognosis than ABCs. ${ }^{1}$

The Papanicolaou test, the most useful screening method for detecting precursor lesions of the uterine cervix, has been used universally with very high diagnostic accuracy. We have been using the Bethesda System revised in 2001 for diagnostic terminology. For evaluation of precancerous and malignant lesions, the diagnostic terms are grouped into squamous cells and glandular cells. However, the system does not classify glandular cell lesions more specifically. Because the incidence of both malignant lesions is very low, distinguishing ACCs and ABCs from other malignancies usually relies on cytology. These tumors are occasionally associated with conventional squamous cell carcinoma, and cytologic findings are similar to those of endometrial cells and masquerade as squamous cell carcinoma. In addition, the diagnostic cytology of ACC is usually negative because of intact overlying mucosa. ${ }^{3}$

In this study, we evaluated the cytological differences between $A C C$ and $A B C$ in order to identify useful cytologic features that can distinguish the two.

\section{MATERIALS AND METHODS}

Cytologic and surgical specimens were obtained from nine patients diagnosed with ACC or ABC from 1998 to 2014 at Gachon University Gil Medical Center. Of the 2,229 cases of uterine cervix carcinomas during this period, there were four cases of ACC $(0.17 \%)$ and five cases of ABC $(0.22 \%)$. All cytology 
specimens were obtained by the routine Papanicolaou cervicovaginal smear and the cases were confirmed by surgical specimens obtained from punch biopsy, conization or hysterectomy. Of the four of ACC cases, one case that coexisted with squamous cell carcinoma. The age range of these ACC cases was 45 to 76 years old at the time of diagnosis. One ACC case was excluded because there were no visible malignant cells on the reviewed cytologic slide. Of the five cases diagnosed as ABCs, four cases coexisted with squamous cell carcinoma. The age range of these $\mathrm{ABC}$ cases was 56 to 78 years old at the time of diagnosis (Table 1).

All patients underwent several cervicovaginal smear tests before and after surgical treatment. Conventional Papanicolaou smear and liquid based preparation methods were used. Of the ACC patients, three had undergone conventional smears, and the one case that was excluded had undergone a liquid based preparation. All patients with $\mathrm{ABC}$, had undergone conventional smear. Cytologic diagnosis was made based on the Bethesda system. After cytological diagnosis, patients underwent surgical procedure for definitive diagnosis or treatment. Punch biopsy, curettage, conization or hysterectomy were performed. We retrospectively reviewed the cervicovaginal smear slides obtained before surgery after histological confirmation.

In all cytology slides, selected individually dispersed representative tumor cells were measured using an image analyzer. A DP70 digital camera (Olympus, Tokyo, Japan) was used to take pictures of tumor cells. I-Solution ver. 8.4 (IMT i-Solution, Coquitlam, BC, Canada) was used for measurement of the area, circumference, major axis, and minor axis of nuclei. These parameters were then used to compare the morphological differentiation of the nuclei of the ACC and ABC in cytology specimens.

Data analysis was performed using SPSS ver. 21.0 (IBM Corp., Armonk, NY, USA). Mann-Whitney U-test was used for comparison of numeric data of area, circumference, major axis, and minor axis of the nuclei of ACC and ABC. Differences between $A C C$ and $A B C$ were considered statistically significant when p-values were less than .05.

\section{RESULTS}

\section{Cytologic characteristics of ACC}

Three cases in this study were ACC. Initial cytologic diagnoses varied, including squamous cell carcinoma, adenocarcinoma, and other malignancies (Table 1). The overall cytologic features of the three cases included large amount of necrotic debris and tumor diathesis as well as numerous orangeophilic globules in the background. Most tumor cells were arranged in three-dimensional overlapping tumor cell clusters, consisting of 40 to 50 tumor cells. The tumor cells occasionally formed glandular structures (Fig. 1A). Rarely, opaque orangeophilic contents were observed in the center of the glandular pattern (Fig. 1B). A few tumor cells were scattered individually. Tumor cells were small and round to oval shape with moderate size variation. Each cell had an indistinct cell border and scant eosinophilic cytoplasm. The nuclei were angulated and hyperchromatic, with coarse granular chromatin pattern. They also had inconspicuous nucleoli (Table 2, Fig. 1C).

Diagnoses of ACCs were confirmed by surgical specimens. One case was cervical punch biopsy, while the others were radical hysterectomy specimens. All cases showed typical histologic findings. There were numerous well circumscribed cellular nests of various sizes in the cervical stroma. Tumors were composed of basaloid cells with indistinct nucleoli arranged in nests with a cribriform appearance, which sometimes had a palisading arrangement in the periphery (Fig. 1D).

\section{Cytologic characteristics of $A B C$}

There were four cases of $A B C$ in this study. All four cases (cases Nos. 4-6 and 8) coexisted with squamous cell carcinomas confirmed by surgical specimens. All but one case (case No. 8) were diagnosed as squamous cell carcinoma in cytologic exami-

Table 1. Clinical summary of adenoid cystic carcinoma and adenoid basal carcinoma

\begin{tabular}{cccccc}
\hline Case No. & Age $(\mathrm{yr})$ & Cytologic diagnosis & Surgical procedure & Histologic diagnosis & Combined lesion \\
\hline 1 & 76 & SCC & Punch biopsy & ACC & - \\
2 & 45 & AC & Hysterectomy & ACC & SD \\
3 & 68 & MN & Hysterectomy & ACC & ABC \\
4 & 78 & SCC, AC & Punch biopsy & ABC & SCC \\
5 & 62 & SCC & Hysterectomy & ABC & SCC \\
6 & 56 & SCC & Hysterectomy & ABC & - \\
7 & 62 & SCC & Hysterectomy & ABC & SCC \\
\hline
\end{tabular}

SCC, squamous cell carcinoma; ACC, adenoid cystic carcinoma; AC, adenocarcinoma; MN, other malignant neoplasm; SD, squamous differentiation; ABC, adenoid basal carcinoma; ASCUS, atypical squamous cells of undetermined significance. 
nations. The cytologic diagnosis of case No. 4 was combined carcinoma, i.e., squamous cell carcinoma and adenocarcinoma. Case No. 8 was diagnosed as atypical squamous cells of undetermined significance (Table 1). In reviewed cytology slides of five cases, some clusters of malignant cells showed distinctive features different from those of pure squamous cell carcinoma. Tumor diathesis was prominent in the background. Tumor cells occasionally presented as single cells, but mostly as three-di-
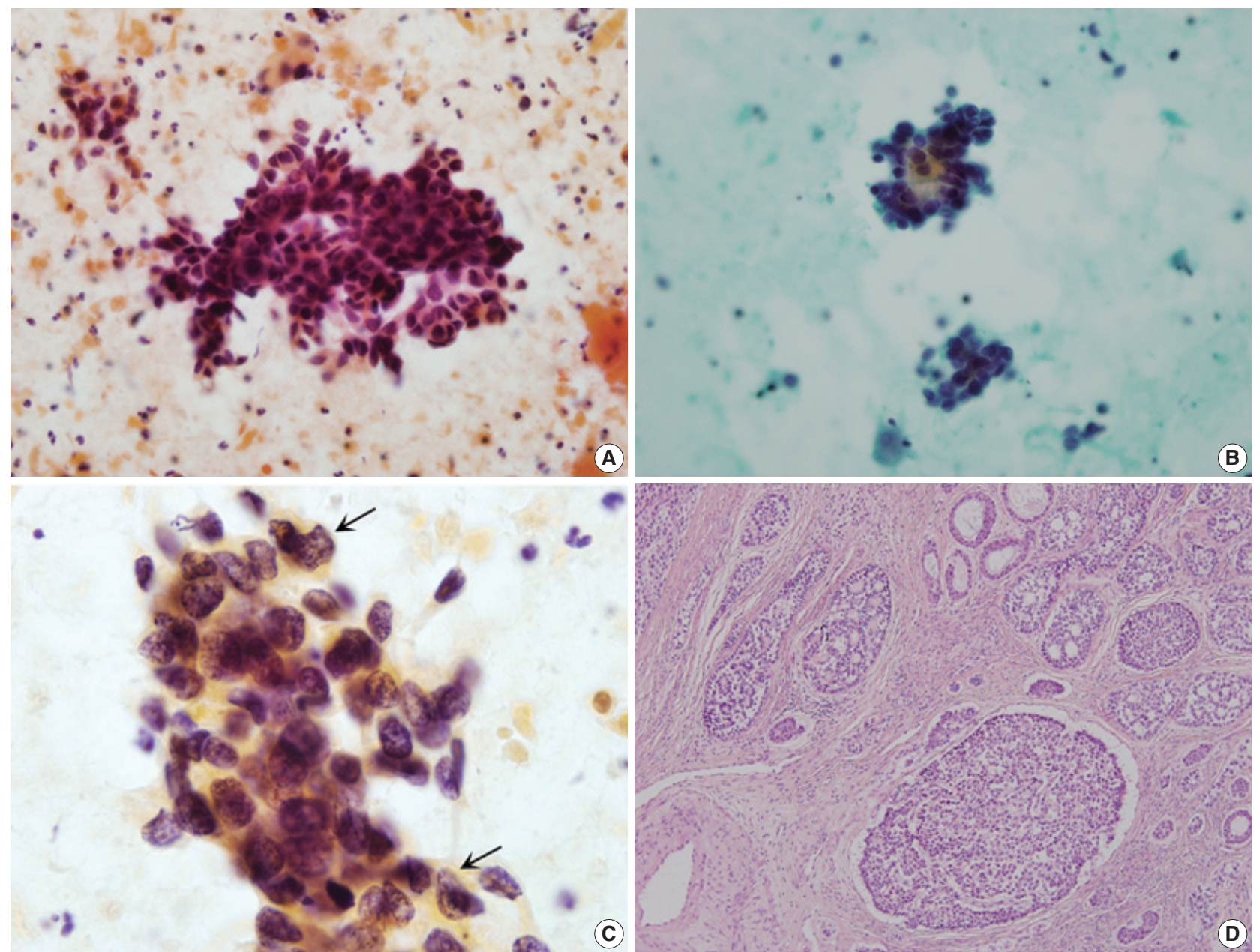

Fig. 1. Cytologic and histopathologic features of adenoid cystic carcinoma. (A) Tumor cells display three-dimensional clusters forming small acini or glandular pattern in necrotic background. (B) Some glandular structures contain opaque orangeophilic contents in the center. (C) The size of nuclei is variable. The nuclei have irregular and angulated membrane with coarsely granular chromatin pattern (arrows). (D) On histologic section, tumor nests consist of basaloid cells forming a cribriform appearance with palisading arrangement in the periphery.

Table 2. Summary of the cytologic features of adenoid cystic carcinoma and adenoid basal carcinoma of the cervix

\begin{tabular}{|c|c|c|}
\hline Cytologic features & Adenoid cystic carcinoma & Adenoid basal carcinoma \\
\hline Background & Bloody and necrotic; orangeophilic globules & Necrotic, mostly \\
\hline Architecture & $\begin{array}{l}\text { Three-dimensional clusters; occasionally glandular structures; } \\
\text { opaque materials in the center of the glands }\end{array}$ & $\begin{array}{l}\text { Three-dimensional clusters of sheets; nuclear overlapping; } \\
\text { rarely peripheral palisading }\end{array}$ \\
\hline $\mathrm{N} / \mathrm{C}$ ratio & Moderate to high & High \\
\hline Nuclear shape & Round to oval & Round to oval \\
\hline Nuclear size $(\mu \mathrm{m})$ & $5-7^{a}$ & $3-4^{\mathrm{a}}$ \\
\hline Nuclear size variation & Mild to moderate ${ }^{b}$ & Mild ${ }^{b}$ \\
\hline Mitotic counts (/100-150 cells) & $2-3$ & $0-1$ \\
\hline Chromatin pattern & Hyperchromatic; coarse granular chromatin pattern & Hyperchromatic; finely granular chromatin pattern \\
\hline Nucleoli & Rarely seen; indistinct, mostly & Rarely seen; small nucleoli, occasionally \\
\hline
\end{tabular}

${ }^{a}$ Estimate based on major axis in our cases; ${ }^{\text {b}}$ Estimate based on major axis with standard deviation in our cases. 

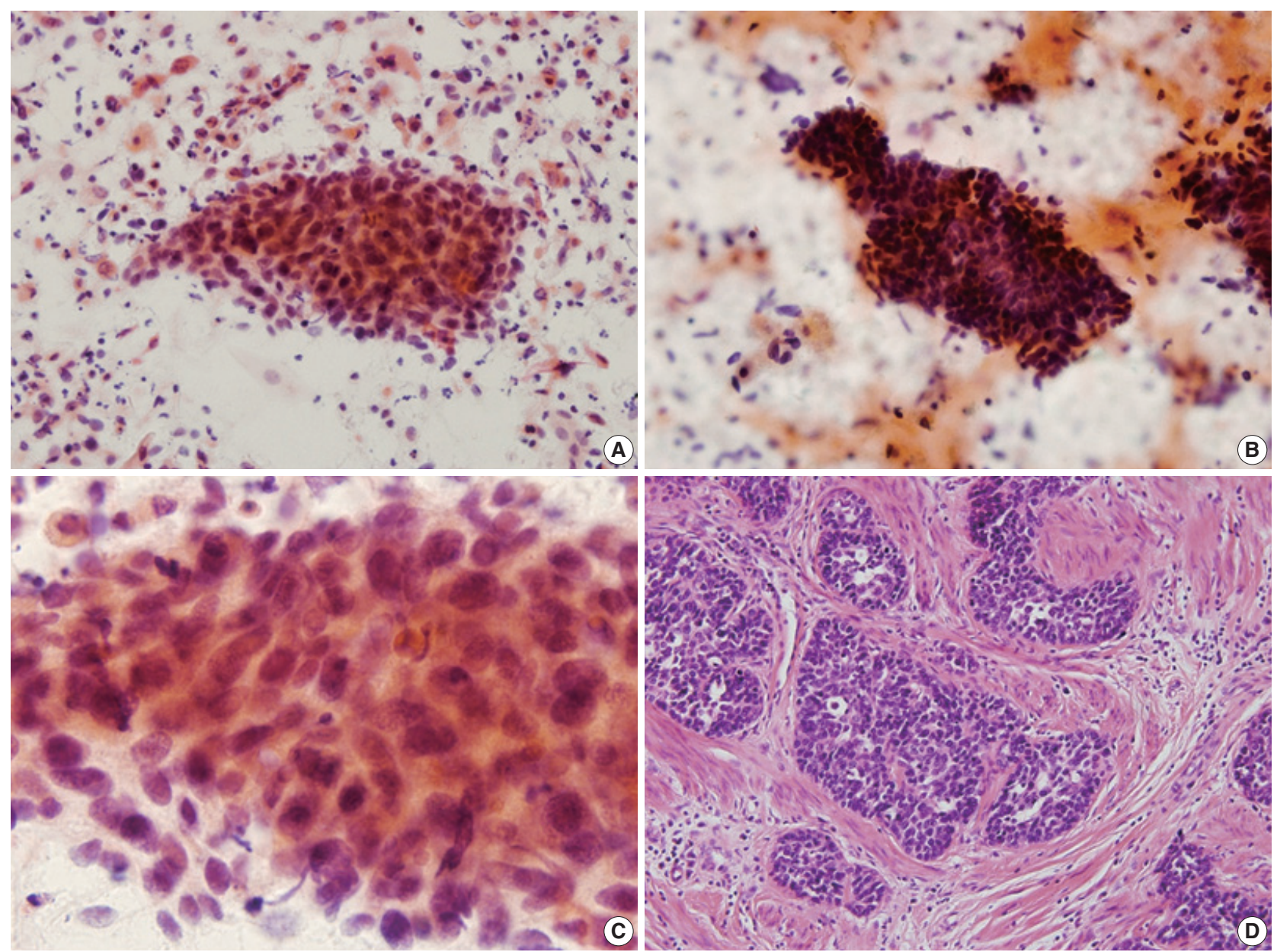

Fig. 2. Cytologic and histopathologic features of adenoid basal carcinoma. (A) Clusters consist of small round basaloid cells in the necrotic background. (B) Tumor cells display three-dimensional clusters with peripheral palisading without glandular pattern. (C) The size of nuclei shows mild variation. The nucleic membrane is smooth with a finely granular chromatin pattern. (D) On histologic section, basaloid cells forming tumor nests display peripheral palisading deep in cervical stroma.

mensional clusters. Rarely, the tumor cell clusters displayed peripheral palisading; however, there was no glandular structure formation (Fig. 2A, B). Individual cells were small and showed slight variation in size with a variable amount of eosinophilic cytoplasm. Nuclei were dense basophilic, hyperchromatic, and sometimes had a fine granular chromatin pattern. Inconspicuous nucleoli were seen (Table 2, Fig. 2C).

Punch biopsy was performed in one case, and radical hysterectomy was performed for cure in the others. Squamous cell carcinomas were also found in four of five cases. All cases showed cervical ulceration grossly, without formation of a mass-like lesion. The tumors had similar histology. $A B C$ was not exposed to the surface. These tumors showed solid tumor cell nests with peripheral palisading or cord like arrangement, and they sometimes formed acini structures without hyaline material. Tumor cells had small basophilic nuclei, with inconspicuous nucleoli
(Fig. 2D).

\section{Analysis of tumor cell nuclear morphology}

The mean ACC nuclear area was $18.556 \pm 8.655 \mu^{2}$, and $11.017 \pm 4.440 \mu^{2}$ in $\mathrm{ABC}$. The mean nuclear perimeter was $23.320 \pm 11.412 \mu \mathrm{m}$ in ACC, and 15.920 $\pm 5.664 \mu \mathrm{m}$ in ABC. The major axis was $5.664 \pm 1.537 \mu \mathrm{m}$ and $4.612 \pm 1.025 \mu \mathrm{m}$ in $A C C$ and $A B C$, respectively. The minor axis was $4.127 \pm 1.107$ $\mu \mathrm{m}$ in ACC, and $3.088 \pm 0.762 \mu \mathrm{m}$ in ABC. Thus, ACC and $A B C$ differed significantly in mean nuclear area, circumference, major axis, and minor axis ( $<$ <.001) (Table 3).

\section{DISCUSSION}

$\mathrm{ACC}$ and $\mathrm{ABC}$ are rare, accounting for less than $1 \%$ of all tumors of the uterine cervix. ${ }^{2}$ ACC of the cervix was first de- 
Table 3. Comparison of nuclear features between adenoid cystic carcinoma and adenoid basal carcinoma

\begin{tabular}{lcrr}
\hline & ACC $(n=117)$ & ABC $(n=218)$ & $p$-value \\
\hline Area $\left(\mu m^{2}\right)$ & $18.556 \pm 8.655$ & $11.017 \pm 4.440$ & $<.001$ \\
Circumference $(\mu \mathrm{m})$ & $23.320 \pm 11.412$ & $15.920 \pm 5.664$ & $<.001$ \\
Major axis $(\mu \mathrm{m})$ & $5.664 \pm 1.537$ & $4.612 \pm 1.025$ & $<.001$ \\
Minor axis $(\mu \mathrm{m})$ & $4.127 \pm 1.107$ & $3.088 \pm 0.762$ & $<.001$ \\
\hline
\end{tabular}

Values are presented as mean \pm standard deviation.

ACC, adenoid cystic carcinoma; ABC, adenoid basal carcinoma.

${ }^{a}$ Statistical significance was determined by Mann-Whitney U-test.

Table 4. Differential diagnosis of adenoid cystic carcinoma and adenoid basal carcinoma $a^{7,13}$

\begin{tabular}{|c|c|c|c|}
\hline Differential diagnosis & Nuclear features & Cytologic features & Structures \\
\hline SCC, keratinizing & $\begin{array}{l}\text { Marked variation in size and shape; irregular } \\
\text { nuclear membrane; coarsely granular and } \\
\text { irregularly distributed chromatin }\end{array}$ & Dense orangiophilic & $\begin{array}{l}\text { Low cellularity; single cells and less } \\
\text { commonly in aggregates }\end{array}$ \\
\hline SCC, nonkeratinizing & $\begin{array}{l}\text { Smaller than HSIL; markedly irregular } \\
\text { distributed chromatin }\end{array}$ & Basophilic & Single or aggregated cells \\
\hline Small cell carcinoma & $\begin{array}{l}\text { Small, relatively uniform cells; hyperchromatic } \\
\text { with granular or stippled chromatin; } \\
\text { frequent mitosis; inconspicuous nucleoli }\end{array}$ & Scant cyanophilic cytoplasm & $\begin{array}{l}\text { Singly and in groups with nuclear } \\
\text { molding }\end{array}$ \\
\hline Endocervical adenocarcinoma & $\begin{array}{l}\text { Columnar configuration enlarged, } \\
\text { pleomorphic nuclei; irregular nuclear } \\
\text { membrane; macronucleoli }\end{array}$ & Usually finely vacuolated & $\begin{array}{l}\text { Abundant abnormal cells; single } \\
\text { cells or two-/three-dimensional } \\
\text { sheets; discohesive groups }\end{array}$ \\
\hline Endometrial carcinoma & $\begin{array}{l}\text { Variation of nuclear size; loss of nuclear } \\
\text { polarity; moderate hyperchromasia; } \\
\text { large with clumped chromatin; } \\
\text { small to prominent nucleoli }\end{array}$ & $\begin{array}{l}\text { Scant, cyanophilic, often } \\
\text { vacuolated; commonly } \\
\text { intracytoplasmic neutrophil }\end{array}$ & Single or small, tight clusters \\
\hline $\begin{array}{l}\text { Benign endometrial } \\
\text { stromal cells }\end{array}$ & $\begin{array}{l}\text { Small round to oval shape; inconspicuous } \\
\text { nucleoli }\end{array}$ & $\begin{array}{l}\text { Scant, basophilic, or occasionally } \\
\text { vacuolated }\end{array}$ & $\begin{array}{l}\text { Three-dimensional ball-like clusters; } \\
\text { double-contoured cluster (the first } \\
\text { half of the menstrual cycle) }\end{array}$ \\
\hline Atrophy & $\begin{array}{l}\text { Intermediate cells with normochromatin; } \\
\text { elongated nuclei; mild hyperchromasia }\end{array}$ & - & Flat, monolayer sheets \\
\hline
\end{tabular}

SCC, squamous cell carcinoma; HSIL, high grade intraepithelial lesion.

scribed by Paalman and Counseller in $1949,{ }^{4}$ while ABC of the cervix was first reported as a separate entity in 1966 by Baggish and Woodruff. ${ }^{5}$ While there have been many single or multiple case reports of ACCs and ABCs, few have been reported on the cytologic characteristics of these two rare malignant tumors of the cervix. ${ }^{3,6-10}$ While some have posited that these tumors share origin from pluripotent reserve cells, ${ }^{11}$ their biologic behavior is quite different: whereas $\mathrm{ABC}$ s are low-grade tumors with good prognosis, ACCs are aggressive, with frequent local recurrences or metastasis. Therefore, accurate differential diagnosis of these two tumors on cervicovaginal smear is very important. However, to date, there are no reports describing the morphologic differences between $\mathrm{ACC}$ and $\mathrm{ABC}$ that allow for sufficient diagnosis by cytology.

All reviewed cervicovaginal smear specimens have numerous three-dimensional overlapping tumor cell clusters. These patterns were described in a report by Grafton et al. ${ }^{8}$ in which three cases were found, and these overlapping tumor cells were said to resemble endometrial adenocarcinoma cells. In the present study, we found that tumor cells in cases of ACC display typical glandular structures, which are hardly seen in cases of ABC? This feature was previously mentioned as acinus formation, cribriform pattern, or rosette-like group. ${ }^{3,6,8,10}$ The cytologic diagnosis of four of the five $\mathrm{ABC}$ cases in this study was squamous cell carcinoma. This is not surprising because squamous cell carcinoma or squamous intraepithelial lesions are commonly associated with $\mathrm{ABC}$. One case was diagnosed as atypical squamous cells of undetermined significance. In some reported $A B C$ cases, the cytologic diagnosis was negative or squamous intraepithelial lesion because of the intact surface epithelium with or without dysplasia or no connection with the epithelium. ${ }^{9}$ While necrosis has not been reported as a feature of $\mathrm{ABC},{ }^{9}$ necrosis was frequently seen in the cervicovaginal smears of our $A B C$ cases, which can to be explained by the coexisting squamous cell carcinoma. Tumor cell clusters showing peripheral palisading in $\mathrm{ABC}$ was a characteristic finding in our cases and other reports. ${ }^{12}$ Glandular structures were found more often in ACC, which have also been mentioned in previous reports as specific find- 
ings of ACC. ${ }^{3,67,7,10}$ While these features are meaningful findings in the two different tumor groups, because ACC and ABC may show similar findings in the histology of surgical specimens, these are not significantly distinguishing points. In our ACC cases, glandular structures had opaque orangeophilc content in the lumina, which may be a helpful feature in making a diagnosis of ACC on the cervicovaginal smear.

The two different tumors in this study showed similar nucleic characteristics including hyperchromasia and indistinct nucleoli. However, we noted that ACC and ABC have different chromatin patterns. Whereas ACC had a coarsely granular chromatin pattern, $A B C$ was more finely granular. In addition, $A C C$ shows a more irregular and angulated nuclear outline than $A B C$ (Figs. 1C, 2C). ${ }^{14}$ Importantly, the nucleic characteristics in ACC and $\mathrm{ABC}$ were significantly different in area, perimeter, major axis, and minor axis. The ratio of minor axis to major axis was approximately 0.728 in ACC and 0.669 in $A B C$, which suggests that the shape of the nuclei is very similar (i.e., oval rather than spherical in both tumors). However, the nucleic area of ACC was 1.684 times larger than that of ABC. Therefore, careful observation of nuclear size, outline, and chromatin pattern may be useful in reaching correct diagnosis when pathologists confuse ACC with ABC.

The differential diagnosis of uterine cervix tumor is very important. Because ACC and ABC are rare tumors, it is difficult to consider these tumors as part of a differential diagnosis. The most misdiagnosed tumor is squamous cell carcinoma, which commonly occurs in the cervix and is coexistent with $A B C$ in many cases. In our institution, the initial cytologic diagnosis was squamous cell carcinoma for five of the eight cases in this study. Three of these five were $\mathrm{ABC}$ associated with squamous cell carcinoma. The cytologic characteristics of squamous cell carcinoma are tumor diathesis in the background, low cellularity with single cells or less commonly aggregated cells. The nuclei show marked variation in size and shape with irregular nuclear membrane and coarsely granular chromatin pattern. However, ACC and ABC commonly show three-dimensional tumor cell clusters and have relatively small, uniform tumor cells. These distinct features could aid pathologists in making a correct diagnosis of $\mathrm{ACC}$ or $\mathrm{ABC}$. Other lesions to consider in differential diagnosis are summarized in Table 4. These include small cell carcinoma, endocervical adenocarcinoma, endometrial adenocarcinoma, benign endometrial cells, and benign cervical lesion such as atrophy (Table 4).

The purpose of this study was to provide comprehensive information on the cytologic characteristics of ACC and $\mathrm{ABC}$ of the cervix, so that pathologists may consider these two malignant tumors when the cervicovaginal smear shows small round cell clusters. Although they show significant difference in nuclear area, circumference, major axis, and minor axis, such difference is not of practical use. However, the size difference of nuclei may be useful. In order to distinguish ACC from $A B C$, to summarize, pathologists will need to make careful observation on structure of tumor cell cluster, nuclear size, irregularity of nuclear membrane, and nuclear chromatin pattern, with a possibility of combined lesions in mind.

\section{Conflicts of Interest}

No potential conflict of interest relevant to this article was reported.

\section{REFERENCES}

1. Kurman RJ, Carcangiu ML, Herrington CS, Young RH. WHO classification of tumours of female reproductive organs. 4th ed. Lyon: IARC Press, 2014.

2. Kurman RJ, Ellenson LH, Ronnett BM. Blaustein's pathology of the female genital tract. 6th ed. Chicago: Springer, 2010; 288-9.

3. Ha SY, Cho H, Oh YH, Lyu GS. The cytologic features of adenoid cystic carcinoma of the uterine cervix: a case report. Korean J Cytopathol 1998; 9: 207-12.

4. Paalman RJ, Counseller VS. Clyindroma of the cervix with procidentia. Am J Obstet Gynecol 1949; 58: 184-7.

5. Baggish MS, Woodruff JD. Adenoid-basal carcinoma of the cervix. Obstet Gynecol 1966; 28: 213-8.

6. Barisic A, Mahovlic V, Ovanin-Rakic A, et al. Cytologic characteristics of adenoid cystic carcinoma of the cervix uteri: case report. Coll Antropol 2010; 34: 233-5.

7. Dayton V, Henry M, Stanley MW, Carson L, Taber E. Adenoid cystic carcinoma of the uterine cervix: cytologic features. Acta Cytol 1990; 34: 125-8.

8. Grafton WD, Kamm RC, Cowley LH. Cytologic characteristics of adenoid cystic carcinoma of the cervix uteri. Acta Cytol 1976; 20: $164-6$.

9. Powers CN, Stastny JF, Frable WJ. Adenoid basal carcinoma of the cervix: a potential pitfall in cervicovaginal cytology. Diagn Cytopathol 1996; 14: 172-7.

10. Ravinsky E, Safneck JR, Chantziantoniou N. Cytologic features of primary adenoid cystic carcinoma of the uterine cervix: a case report. Acta Cytol 1996; 40: 1304-8.

11. Grayson W, Taylor LF, Cooper K. Adenoid cystic and adenoid basal carcinoma of the uterine cervix: comparative morphologic, mucin, 
and immunohistochemical profile of two rare neoplasms of putative 'reserve cell' origin. Am J Surg Pathol 1999; 23: 448-58.

12. Peterson LS, Neumann AA. Cytologic features of adenoid basal carcinoma of the uterine cervix: a case report. Acta Cytol 1995; 39: 563-8.

13. Solomon D, Nayer R. The Bethesda system for reporting cervical cytology: definitions, criteria, and explanatory notes. 2nd ed. New York: Springer, 2003.

14. Russell MJ, Fadare O. Adenoid basal lesions of the uterine cervix: evolving terminology and clinicopathological concepts. Diagn Pathol 2006; 1: 18. 